\title{
Cubature Formulas of Degree Nine for Symmetric Planar Regions
}

\author{
By Robert Piessens and Ann Haegemans*
}

\begin{abstract}
A method of constructing 19-point cubature formulas with degree of exactness 9 is given for two-dimensional regions and weight functions which are symmetric in each variable. For some regions, e.g., the square and the circle, these formulas can be reduced to 18-point formulas.
\end{abstract}

1. Introduction. We consider cubature formulas,

$$
\iint_{R} w(x, y) f(x, y) d x d y \cong \sum_{k=1}^{N} w_{k} f\left(x_{k}, y_{k}\right),
$$

which are exact for all polynomials in $x$ and $y$ of degree $\leqslant d$ but not for all polynomials of degree $d+1$. Such formulas are said to have degree $d$.

According to Rabinowitz and Richter [1], we say that formula (1) is a 'good' formula if it has all of its points $\left(x_{k}, y_{k}\right)$ inside the region $R$ and all of its coefficients $w_{k}$ positive. We assume that $R$ is a symmetric region (i.e., $(x, y) \in R$ implies $( \pm x, \pm y$ ) $\in R)$ and that $w(x, y)$ is symmetric in $x$ and $y$ and nonnegative. In several recent publications [1] $-[5]$, cubature formulas are computed which have the minimum number of points for their degree. Minimum-point formulas are closely connected with the theory of orthogonal polynomials [6]. This theory, however, is not yet sufficiently developed to give practical results in the case of high degree of exactness $(d>7)$. For $9 \leqslant d \leqslant 15$, Rabinowitz and Richter [1] have computed perfectly symmetric formulas, by solving a system of nonlinear equations. For $d=9$, the number of points in their formulas is $N=20$.

Until now, no formulas of degree 9 with less than 20 points were known for the square; for the circle, only one 19-point formula is computed by Albrecht [7]. In this note, we describe a numerical method for the construction of 18- and 19-point formulas, if they exist.

It is very likely, but not proved, that for the square and the circular domain with weight function $w(x) \equiv 1$ the 18-point formulas constructed in this way are minimumpoint formulas.

2. Method of Construction. Since the region and the weight function are symmetric, it is reasonable io consider only symmetric formulas. Firstly, we construct 19-point formulas. As we shall show further, these formulas must have at least 12 points $\left(x_{k}, y_{k}\right)$ with $x_{k} \neq 0$ and $y_{k} \neq 0$. We consider then the formula,

Received June 27, 1974.

AMS (MOS) subject classifications (1970). Primary 41A55, 41A63, 65D30; Secondary 41 A10.

Key words and phrases. Approximate integration, cubature formula, degree of exactness, planar region, orthogonal polynomials.

*Stagiair of the NFWO (Belgium). 
$\iint_{R} w(x, y) f(x, y) d x d y$

$$
\begin{aligned}
\cong & \sum_{k=1}^{3} w_{k}\left[f\left(x_{k}, y_{k}\right)+f\left(-x_{k}, y_{k}\right)+f\left(x_{k},-y_{k}\right)+f\left(-x_{k},-y_{k}\right)\right] \\
& +w_{4}\left[f\left(x_{4}, 0\right)+f\left(-x_{4}, 0\right)\right]+\sum_{k=5}^{6} w_{k}\left[f\left(0, y_{k}\right)+f\left(0,-y_{k}\right)\right]+w_{7} f(0,0),
\end{aligned}
$$

where $x_{k}>0$ and $y_{k}>0$.

For the computation of the coefficients $w_{k}$ and the points $\left(x_{k}, y_{k}\right)$ of (2), we have the following set of equations

$$
4 \sum_{k=1}^{3} w_{k} x_{k}^{2 \alpha} y_{k}^{2 \beta}=m_{2 \alpha, 2 \beta}, \quad 0<\alpha+\beta \leqslant 4,
$$

$$
4 \sum_{k=1}^{3} w_{k} x_{k}^{2 \alpha}+2 w_{4} x_{4}^{2 \alpha}=m_{2 \alpha, 0}, \quad 0<\alpha \leqslant 4,
$$

$$
4 \sum_{k=1}^{3} w_{k} y_{k}^{2 \beta}+2 \sum_{k=5}^{6} w_{k} y_{k}^{2 \beta}=m_{0,2 \beta}, \quad 0<\beta \leqslant 4,
$$

$$
4 \sum_{k=1}^{3} w_{k}+2 \sum_{k=4}^{6} w_{k}+w_{7}=m_{0,0} .
$$

Here $\alpha$ and $\beta$ are natural numbers and

$$
m_{\alpha, \beta}=\iint_{R} w(x, y) x^{\alpha} y^{\beta} d x d y .
$$

Firstly, we choose $x_{2}, x_{3}$ and $y_{3}$ arbitrarily. The system of six equations (3.a) can then be solved explicitly

(4)

$$
\begin{gathered}
w_{3}=\frac{\left(m_{2,4}^{2} m_{6,2}+m_{4,4}^{2} m_{2,2}-2 m_{4,4} m_{2,4} m_{4,2}-m_{6,2} m_{2,2} m_{2,6}+m_{4,2}^{2} m_{2,6}\right)}{\left\{\left[\left(m_{2,4}^{2}-m_{2,2} m_{2,6}\right) x_{3}^{4}+\left(m_{4,2}^{2}-m_{6,2} m_{2,2}\right) y_{3}^{4}\right.\right.} \\
+2\left(m_{4,2} m_{2,6}-m_{4,4} m_{2,4}\right) x_{3}^{2}+2\left(m_{2,4} m_{6,2}-m_{4,4} m_{4,2}\right) y_{3}^{2} \\
\left.\left.+2\left(m_{4,4} m_{2,2}-m_{2,4} m_{4,2}\right) x_{3}^{2} y_{3}^{2}+m_{4,4}^{2}-m_{6,2} m_{2,6}\right] 4 x_{3}^{2} y_{3}^{2}\right\}, \\
x_{1}^{2}=\left(m_{4,2}^{*} x_{2}^{2}-m_{6,2}^{*}\right) /\left(m_{2,2}^{*} x_{2}^{2}-m_{4,2}^{*}\right), \\
y_{1}^{2}=\left(m_{2,4}^{*} x_{2}^{2}-m_{4,4}^{*}\right) /\left(m_{2,2}^{*} x_{2}^{2}-m_{4,2}^{*}\right), \\
y_{2}^{2}=\left[\left(m_{4,4}^{*} m_{2,2}^{*}-m_{2,4}^{*} m_{4,2}^{*}\right) x_{2}^{2}+m_{2,4}^{*} m_{6,2}^{*}-m_{4,4}^{*} m_{4,2}^{*}\right] /\left(m_{6,2}^{*} m_{2,2}^{*}-m_{4,2}^{*}{ }^{2}\right), \\
w_{1}=\left(m_{2,2}^{*} x_{2}^{2}-m_{4,2}^{*}\right)^{2} /\left[4 x_{1}^{2} y_{1}^{2}\left(m_{2,2}^{*} x_{2}^{4}-2 m_{4,2}^{*} x_{2}+m_{6,2}^{*}\right)\right], \\
w_{2}=\left(m_{6,2}^{*} m_{2,2}^{*}-m_{4,2}^{*}\right) /\left[4 x_{1}^{2} y_{1}^{2}\left(m_{2,2}^{*} x_{2}^{4}-2 m_{4,2}^{*} x_{2}+m_{6,2}^{*}\right)\right],
\end{gathered}
$$


where $m_{\alpha, \beta}^{*}=m_{\alpha, \beta}-4 w_{3}^{2} x_{3}^{\alpha} y_{3}^{\alpha}$. Formula (4) shows that the coefficient $w_{3}$ corresponding to the arbitrarily chosen point $\left(x_{3}, y_{3}\right)$ is independent of the position of the other points and also different from zero (except perhaps for exceptional regions or weight functions). This means that a symmetric formula of degree 9 requires at least three points in each quadrant of the region.

We consider now the set of four equations (3.b) into which we substitute the free parameters $x_{2}, x_{3}$ and $y_{3}$ and the computed values $w_{1}, x_{1}, y_{1}, w_{2}$ and $y_{3}$. The first two of these equations can be used for the computation of $w_{4}$ and $x_{4}$. The remaining two equations of this set are then considered as a system of two simultaneous equations in the unknowns $x_{2}, x_{3}$ and $y_{3}$. This will generally leave one free parameter, say $y_{3}$. The system of nonlinear equations in the unknowns $x_{2}$ and $x_{3}$ must be solved numerically.

The parameters $y_{5}, y_{6}, w_{5}$ and $w_{6}$ are then computed by solving the system (3.c). The numbers $y_{5}^{2}$ and $y_{6}^{2}$ are the roots of the quadratic equation,

$$
\left(m_{0,4}^{*}{ }^{2}-m_{0,6}^{*} m_{0,2}^{*}\right) z^{2}+\left(m_{0,2}^{*} m_{0,8}^{*}-m_{0,4}^{*} m_{0,6}^{*}\right) z+\left(m_{0,6}^{*}{ }^{2}-m_{0,4}^{*} m_{0,8}^{*}\right)=0 \text {, }
$$

while

$$
w_{5}=\left(m_{0,2}^{*} y_{6}^{2}-m_{0,4}^{*}\right) /\left[2 y_{5}^{2}\left(y_{6}^{2}-y_{5}^{2}\right)\right]
$$

and

$$
w_{6}=\left(m_{0,4}^{*}-m_{0,2}^{*} y_{5}^{2}\right) /\left[2 y_{6}^{2}\left(y_{6}^{2}-y_{5}^{2}\right)\right]
$$

where

$$
m_{0, \beta}^{*}=m_{0, \beta}-4 \sum_{k=1}^{3} w_{k} y_{k}^{\beta} .
$$

Finally, $w_{7}$ is computed from (3.d).

From this method of solution, we conclude that there are generally more solutions, since $y_{3}$ is still a free parameter. However, it is not impossible that several or even all solutions are complex-valued. In this last case, a 19-point formula of the form (2) does not exist. However, there may still exist a formula of the form,

$$
\begin{aligned}
& \iint_{R} w(x, y) f(x, y) d x d y \\
& \cong \sum_{k=1}^{4} w_{k}\left[f\left(x_{k}, y_{k}\right)+f\left(-x_{k}, y_{k}\right)+f\left(x_{k}-y_{k}\right)+f\left(-x_{k},-y_{k}\right)\right] \\
&+w_{5}\left[f\left(x_{5}, 0\right)+f\left(-x_{5}, 0\right)\right]+w_{6} f(0,0) .
\end{aligned}
$$

In order to obtain an 18-point formula of degree 9 , we consider $w_{7}$ as a function of $y_{3}$ and we solve the equation $w_{7}\left(y_{3}\right)=0$ (if there exists a real-valued solution). We conjecture that the 18-point formulas computed in this way are minimum-point formulas.

For several regions and weight functions, we have carried out numerical experiments. We summarize the most important results in the following section.

3. Some Results. (i) For the square $R=C_{2}=\{(x, y):-1 \leqslant x, y \leqslant 1\}$ and $w(x, y) \equiv 1$ infinitely many 'good' 19-point formulas of degree 9 exist. There are also 
at least two 'good' 18-point formulas, the parameters of which we give in Tables 1 and 2 to 20 significant digits. The points of both formulas are common zeros of three orthogonal polynomials of degree 5. These orthogonal polynomials are $P_{0}+\lambda_{1} P_{2}$, $P_{0}+\lambda_{2} P_{4}$ and $P_{1}+\mu_{1} P_{3}+\mu_{2} P_{5}$ where, for the first formula,

$$
\begin{array}{ll}
\lambda_{1}=0.24819696, & \lambda_{2}=0.25574007, \\
\mu_{1}=0.00469095, & \mu_{2}=-0.96079906,
\end{array}
$$

and for the second formula,

and where

$$
\begin{array}{ll}
\lambda_{1}=-0.22003380, & \lambda_{2}=-0.30398642, \\
\mu_{1}=-0.33809909, & \mu_{2}=-1.33579356,
\end{array}
$$

$$
\begin{aligned}
& P_{0}(x, y)=x^{5}-10 x^{3} / 9+5 x / 21, \\
& P_{1}(x, y)=x^{4} y-6 x^{2} y / 7+3 y / 35, \\
& P_{2}(x, y)=x^{3} y^{2}-x^{3} / 3-3 x y^{2} / 5+x / 5, \\
& P_{3}(x, y)=P_{2}(y, x), \\
& P_{4}(x, y)=P_{1}(y, x), \\
& P_{5}(x, y)=P_{0}(y, x),
\end{aligned}
$$

are the basic orthogonal polynomials for $C_{2}$.

(ii) For the circle $R=S_{2}=\left\{(x, y): x^{2}+y^{2} \leqslant 1\right\}$ with $w(x, y) \equiv 1$, infinitely many 'good' 19-point formulas of degree 9 exist. We have also computed one 18-point formula which has, however, four of its points outside $S_{2}$ (see Table 3). The points of this formula are the common zeros of the orthogonal polynomials $P_{0}+\lambda_{1} P_{2}, P_{0}+$ $\lambda_{2} P_{4}$ and $P_{1}+\mu_{1} P_{3}+\mu_{2} P_{5}$ with

$$
\begin{array}{ll}
\lambda_{1}=0.56685388, & \lambda_{2}=0.16924976, \\
\mu_{1}=0.56433371, & \mu_{2}=-0.65980030,
\end{array}
$$

and where

$$
\begin{aligned}
& P_{0}(x, y)=x^{5}-x^{3}+3 x / 16 \\
& P_{1}(x, y)=x^{4} y-3 x^{2} y / 5+3 y / 80 \\
& P_{2}(x, y)=x^{3} y^{2}-x^{3} / 10-3 x y^{2} / 10+3 x / 80 \\
& P_{3}(x, y)=P_{2}(y, x) \\
& P_{4}(x, y)=P_{1}(y, x) \\
& P_{5}(x, y)=P_{0}(y, x) .
\end{aligned}
$$


(iii) For the entire plane $R=E_{2} r^{2}=\{(x, y):-\infty \leqslant x, y \leqslant \infty\}$ with weight function $w(x, y)=\exp \left(-x^{2}-y^{2}\right)$, infinitely many 'good' 19-point formulas exist. However, we have not found any 18-point formula.

(iv) For the entire plane $R=E_{2}^{r}=\{(x, y)$ : $-\infty \leqslant x, y \leqslant \infty\}$ with weight function $w(x, y)=\exp \left(-\left(x^{2}+y^{2}\right)^{1 / 2}\right)$, we have not found any real solution of the system of equations (3.a, b, c, d).

A number of the 19-point formulas for $C_{2}, S_{2}$ and $E_{2}^{2}$ are tabulated in [8].

TABLE 1. First 18-point formula for the square

\begin{tabular}{|c|c|c|c|}
\hline$k$ & $x_{k}$ & $y_{k}$ & $w_{k}$ \\
\hline 1 & 0.87980721399752853896 & 0.92797961509268528861 & $0.68416522462309305679(-1)$ \\
2 & 0.50445910315479838456 & 0.75347199103161505380 & 0.27903384209687301395 \\
3 & 0.91531235408227324183 & 0.42299357094876513066 & 0.16806533822999587126 \\
4 & 0.57882826011929170546 & 0 & 0.40927359555433144329 \\
5 & 0 & 0.97700090158004246059 & 0.10648011781560231854 \\
6 & 0 & 0.39364057271848893512 & 0.45321488105170985638 \\
\hline
\end{tabular}

TABLE 2. Second 18-point formula for the square

\begin{tabular}{|c|c|c|c|}
\hline$k$ & $x_{k}$ & $y_{k}$ & $w_{k}$ \\
\hline 1 & 0.93742666622066710914 & 0.94145119299928430974 & $0.42853317248897088536(-1)$ \\
2 & 0.57077001686857404415 & 0.79214654516847247531 & 0.25788406360659644304 \\
3 & 0.89774224179848572970 & 0.40001733897633692860 & 0.19397744037003970872 \\
4 & 0.49471787965159623409 & 0 & 0.45212398131214854997 \\
5 & 0 & 0.98085697194664054422 & 0.10243215270991495821 \\
6 & 0 & 0.48311469619727965642 & 0.45601422352687001122 \\
\hline
\end{tabular}

TABLE 3. 18-point formula for the circle

\begin{tabular}{|c|c|c|c|}
\hline$k$ & $x_{k}$ & $y_{k}$ & $w_{k}$ \\
\hline 1 & 0.86686876801492291622 & 0.28376671812094800827 & 0.12937261598422958670 \\
2 & 0.63925306939199114680 & 0.95409639862933054563 & $0.77785540900483355115(-2)$ \\
3 & 0.48645191470776426796 & 0.63982457013387676359 & 0.22713305094453060651 \\
4 & 0.51286789206607718656 & 0 & 0.32090673961381781518 \\
5 & 0 & 0.88859953503035797854 & 0.16042870730308439624 \\
6 & 0 & 0.35335517369353007690 & 0.36089243784037735036 \\
\hline
\end{tabular}

Acknowledgment. This research is supported by the FKFO, Belgium under grant no. 10174.

The computations are carried out on the IBM 370/158 computer of the Computing Centre of the University of Leuven. 
1. P. RABINOWITZ \& N. RICHTER, "Perfectly symmetric two-dimensional integration formulas with minimal number of points," Math. Comp., v. 23, 1969, pp. 765-779. MR 41 \#2928.

2. I. P. MYSOVSKIH, "On the construction of cubature formulas with fewest nodes," Dokl. Akad. Nauk SSSR, v. 178, 1968, pp. 1252-1254 = Soviet Math. Dokl., v. 9, 1968, pp. 277-280. MR 36 \#7328.

3. R. FRANKE, "Minimal point cubatures of precision seven for symmetric regions," SIAM J. Numer. Anal., v. 10, 1971 , pp. 849-882.

4. S. HABER, "Numerical evaluation of multiple integrals," SIAM Rev., v. 12, 1970, pp. 481-526. MR 44 \#2342.

5. A. H. STROUD, Approximate Calculation of Multiple Integrals, Prentice-Hall Ser. in Automatic Computation, Prentice-Hall, Englewood Cliffs, N. J., 1971. MR 48 \#348.

6. A. H. STROUD, "Integration formulas and orthogonal polynomials for two variables," SIAM J. Numer. Anal., v. 6, 1969, pp. 222-229. MR 41 \#6400.

7. J. ALBRECHT, "Formeln zur numerischen Integration über Kreisbereiche," $Z$. Angew. Math. Mech., v. 40, 1960, pp. 514-517. MR 22 \#11514.

8. A. HAEGEMANS \& R. PIESSENS, Tables of Cubature Formulas of Degree Nine for Symmetric Planar Regions. (Report to be published.) 